\title{
Functional Swallowing Outcomes in Transoral Robotic Surgery Patients With and Without Adjuvant Treatment
}

\author{
Caitlin Coviello, MBA ${ }^{1 *}$; Opeoluwa Daniyan, MD; Carlos Green, MD; Mario Landera, SLPD; \\ Donna Lundy, PhD²; Alejandro Mantero, PhD; Jamal Ahmed, MD,4; Giovana Thomas, MD, FACS
}

'Department of Otolaryngology, Division of Head and Neck Surgery, University of Miami, Miami, Florida, USA 2Department of Otolaryngology, Division of Speech Language Pathology, University of Miami, Miami, Florida, USA

${ }^{3}$ Department of Public Health Sciences, Division of Biostatistics, University of Miami, Miami, Florida, USA

${ }^{4}$ University of Wisconsin Hospitals and Clinics, Madison, Wisconsin, USA

\begin{abstract}
Objective: Dysphagia is a common side effect of treatment for head and neck malignancy. Transoral robotic surgery (TORS) is gaining momentum as a minimally invasive treatment option for oropharyngeal squamous cell carcinoma (OPSCC). Although favorable swallowing outcomes have been reported following single modality treatment with TORS, many patients require post-operative adjuvant therapy due to unforeseen negative prognostic factors. Our objective was to evaluate short- and long-term swallowing function in patients with OPSCC undergoing TORS alone, TORS plus radiation therapy (RT), and TORS plus chemoradiation therapy (CRT).

Methods: A retrospective cohort study was conducted between September 2012 through November 2017 in patients who underwent TORS for primary treatment of OPSCC with at least 12 months of follow-up data. Functional swallowing outcomes were measured based on the functional oral intake scale (FOIS). Descriptive statistics were reported, and analysis was performed using a Kruskal-Wallis test.

Results: Sixty patients met criteria. Eleven patients were treated with TORS alone, 12 underwent TORS+RT, and 37 underwent TORS+CRT. All groups showed improved FOIS scores 6 months after treatment and approached normal or near normal FOIS scores 12 months after treatment. Patients who underwent TORS alone showed a trend towards higher FOIS scores when compared to TORS with adjuvant therapy. Patients in the TORS alone group had higher FOIS scores than those in the TORS plus chemoradiation group at 12 months post-treatment $(P=0.055)$.

Conclusion: Dysphagia is an important consequence of head and neck cancer treatment. Using the FOIS, we demonstrated that swallowing function in TORS patients improves within one year. While not statistically significant, our study showed increased FOIS scores in TORS alone patients when compared to TORS plus adjuvant therapy. This study emphasizes the importance of diligent selection in patients undergoing TORS to avoid poor functional swallowing outcomes, particularly in those that may need adjuvant therapy.
\end{abstract}

\section{INTRODUCTION}

Human Papilloma Virus (HPV)-related oropharyngeal squamous cell carcinoma (OPSCC) is rapidly becoming the most commonly diagnosed head and neck malignancy $[1,2]$ and its prevalence is expected to continue to increase in the coming years $[3,4]$. While oropharyngeal cancers have traditionally been treated with radiation therapy, chemoradiation therapy and open surgical treatments including labiotomy and mandibulectomy [5-8], these treatment options often lead to significant functional sequelae that may be long-term [913]. Transoral robotic surgery (TORS) is rapidly gaining momentum as a minimally invasive treatment option for a select patient population as it has been shown to provide sufficient control of oncologic disease $[14,15]$ with superior functional outcomes [16-22].

The advent of TORS technology is particularly important for patients diagnosed with HPV-related OPSCC. HPV-related OPSCC patients are often younger with longer overall life expectancies [23,24]; therefore, avoiding long-term sequelae of therapy has become an important issue in this patient population [25]. Although favorable swallowing outcomes have been reported following single modality treatment with TORS [26], research has shown that many patients require post-operative adjuvant radiation or chemoradiation due to unforeseen negative prognostic factors discovered in their resected tumor or neck specimen [27]. These adjuvant treatments are thought to further impact acute and late toxicities, can result in fibrosis and scarring, and ultimately have negative effects on swallowing safety and efficiency $[25,28]$.

Several studies have investigated swallowing function after TORS with methods involving patient-reported quality of life and function $[16,29]$. In a systematic review of a MEDLINE database, Hutcheson et al. found that feeding tube rates were the most commonly reported measure to assess functional outcome [30]; however, there is a clear lack of published studies utilizing standardized measures to assess functional outcomes and oral intake ability in these patients. The functional oral intake scale (FOIS) is a standardized subjective dietary scale originally validated in stroke patients to assess functional outcomes through a patient's oral intake over time [31]. It has previously been used to assess swallowing and oral intake function in head and neck cancer patients in the pre-treatment period [32] as well as in the post-operative period in head and neck squamous cell carcinoma TORS patients [33]. FOIS is a clinician-rated scale based on a patient's report of oral intake and provides clinicians a better understanding of a patient's functional ability [31].

The purpose of this retrospective study was to assess the impact of three treatment sequences [TORS alone, TORS followed by radiation (RT), and TORS followed by chemoradiation (CRT)] on the short ( 3 and 6 months) and longterm (12 months) functional outcome of oral intake in patients with HPV-related OPSCC using the FOIS. We hypothesized that patients undergoing triple therapy (TORS followed by chemoradiation) would have poorer short and long-term swallowing outcomes. 


\begin{tabular}{|cl|}
\hline \multicolumn{2}{|c|}{ Table 1. Functional Oral Intake Scale } \\
\hline Level & Description \\
\hline 1 & Nothing by mouth \\
\hline 2 & Tube dependent with minimal attempts of food or liquid \\
\hline 3 & Tube dependent with consistent oral intake of food or liquid \\
\hline 4 & Total oral diet of a single consistency \\
\hline 5 & $\begin{array}{l}\text { Total oral diet of multiple consistencies but requiring special } \\
\text { preparation or compensations }\end{array}$ \\
\hline 6 & $\begin{array}{l}\text { Total oral diet of multiple consistencies without special preparation } \\
\text { but with specific food limitations }\end{array}$ \\
\hline 7 & Total oral intake without restrictions \\
\hline
\end{tabular}

${ }^{*}$ Higher levels represent improved oral intake and functional ability.

\section{METHODS}

A retrospective analysis was performed on patients who underwent TORS for primary treatment of OPSCC at the University of Miami Health System from September 2012 to November 2017. Inclusion criteria required that all patients be at least 18 years of age and have FOIS scores available at 3, 6, and 12 months post-treatment. Patients were excluded from the study if they had inadequate medical records or prior treatment for cancers in the head and neck region. Institutional review board approval was granted by the University of Miami for the completion of this study.

\section{Data Collection}

A database was created from review of patient electronic medical records. Demographic data was collected including age at surgery, sex, race, as well as tobacco and alcohol habits. Pathologic data obtained included tumor classification, nodal status, presence of metastasis, HPV status, p16 positivity, and details of adjuvant therapy were recorded. All patients were staged according to 8th edition American Joint Committee on Cancer guidelines [34].

\section{Treatment Regimen}

Patients underwent TORS radical tonsillectomy, oropharyngectomy, or base of tongue resection based on primary tumor location. Resection was performed using the da Vinci Surgical System (Sunnyvale, CA). Neck dissection

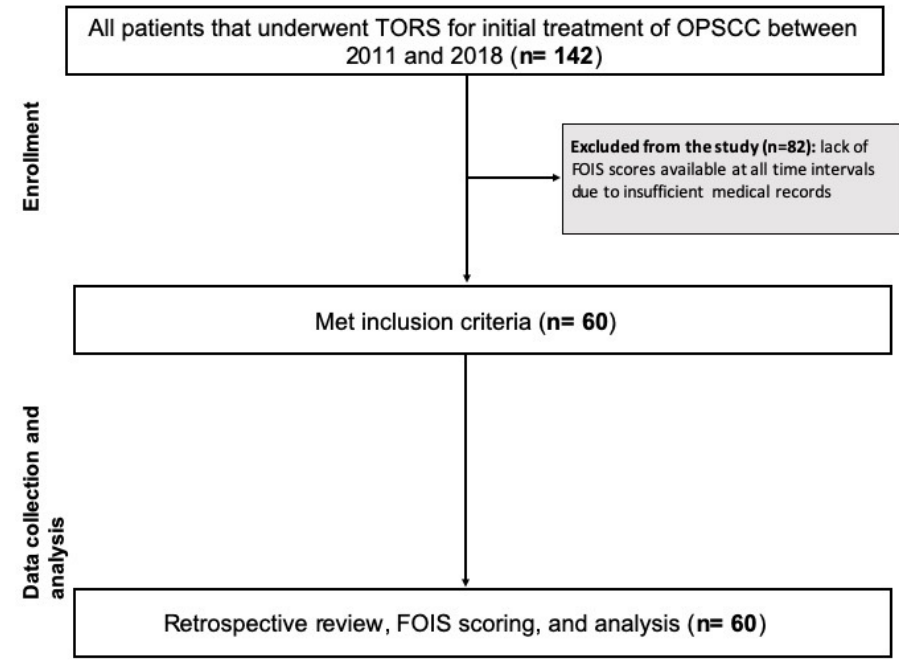

Figure 1. Exclusion and inclusion criteria of study population demonstrating the flow of patient enrollment, data collection, and statistical analysis. FOIS, functional oral intake scale; OPSCC, oropharyngeal squamous cell carcinoma; TORS, transoral robotic surgery. was performed on all patients either at the time of TORS or shortly prior to TORS. Immunohistochemistry was performed based on surgical pathology to assess for HPV DNA and protein 16 (p16) status. In accordance with current National Comprehensive Cancer Network guidelines [35], established clinical and pathological criteria were used to determine candidacy for adjuvant treatment. Briefly, indications for adjuvant RT included extracapsular extension, positive margins, nodal disease in levels IV or V, perineural invasion, vascular embolism, or lymphatic invasion. Indications for CRT included extracapsular extension or positive margins. Patients who were treated with adjuvant RT received 60-66 Gy 5 days per week for 6-6.5 weeks if they had high risk features, such as positive margins and 44-50 Gy to 54-63 Gy if they had low risk features, such as suspected subclinical spread. Patients who received adjuvant CRT received concurrent cisplatin and radiation therapy as listed above.

\section{Functional Outcome Measures}

FOIS is a 7-point dietary scale with higher numbers representing improved oral intake and functional ability (Table 1). Standard institutional protocol at the time of this study for TORS patients was to undergo assessment of functional swallowing outcomes in the post-treatment period for at least 12 months and until maximum swallowing improvement was achieved. This was made possible by way of a multidisciplinary head and neck clinic, which allows for collaboration between head and neck surgeons and speech-language pathologists. Medical records were reviewed to document FOIS scores at 3, 6, and 12 months post treatment based on patient-reported oral intake.

\section{Statistical Analysis}

All statistical analyses were performed by the University of Miami Department of Public Health, Division of Biostatistics. FOIS swallowing outcomes were reported via medians and interquartile ranges with a maximum 12-month follow up. Comparisons were made using Kruskal-Wallis test due to the ordered categorical nature of the measure. Descriptive analysis was used to compare each of the treatment groups with regard to functional swallowing outcomes. All analyses were performed in R version 3.4.1.

\section{RESULTS}

\section{Study Population Characteristics}

One hundred forty-two patients underwent TORS for previously untreated OPSCC at our institution from September 2012 to November 2017. Of these patients, a total of 60 met inclusion criteria. Figure 1 demonstrates a diagram of those patients included in the study. Table 2 summarizes the study population. The majority of our study population were white males with a median age of 63 years. Ninety-three percent of our patients had T1-T2 stage disease and $90 \%$ of our patients were classified as stage 1 OPSCC. No patients had metastatic disease. One patient diagnosed with T2 stage disease clinically was found to have T4 disease pathologically due to skeletal muscle invasion at the base of tongue.

Over $50 \%$ of the study population underwent TORS+CRT $(n=37)$, largely due to extracapsular extension $(n=25)$. All extracapsular extension was found microscopically following surgical intervention. As expected, the TORS+RT and TORS+CRT treatment groups had a higher likelihood of having nodal disease and more advanced disease staging.

\section{FOIS Scores: General Trends}

Figure 2 demonstrates boxplots of FOIS scores at 3, 6, and 12 months post-treatment. Figure $2 \mathrm{~A}$ represents these scores in the TORS alone group, Figure $2 \mathrm{~B}$ in the TORS+RT treatment group, and Figure $2 \mathrm{C}$ in the TORS+CRT treatment group. Median FOIS scores are demonstrated by the horizontal bold line in each of the boxplots with the box representing the interquartile range at each time interval. Overall, there is an improvement in median FOIS scores among all three groups over time. The majority of patients demon- 

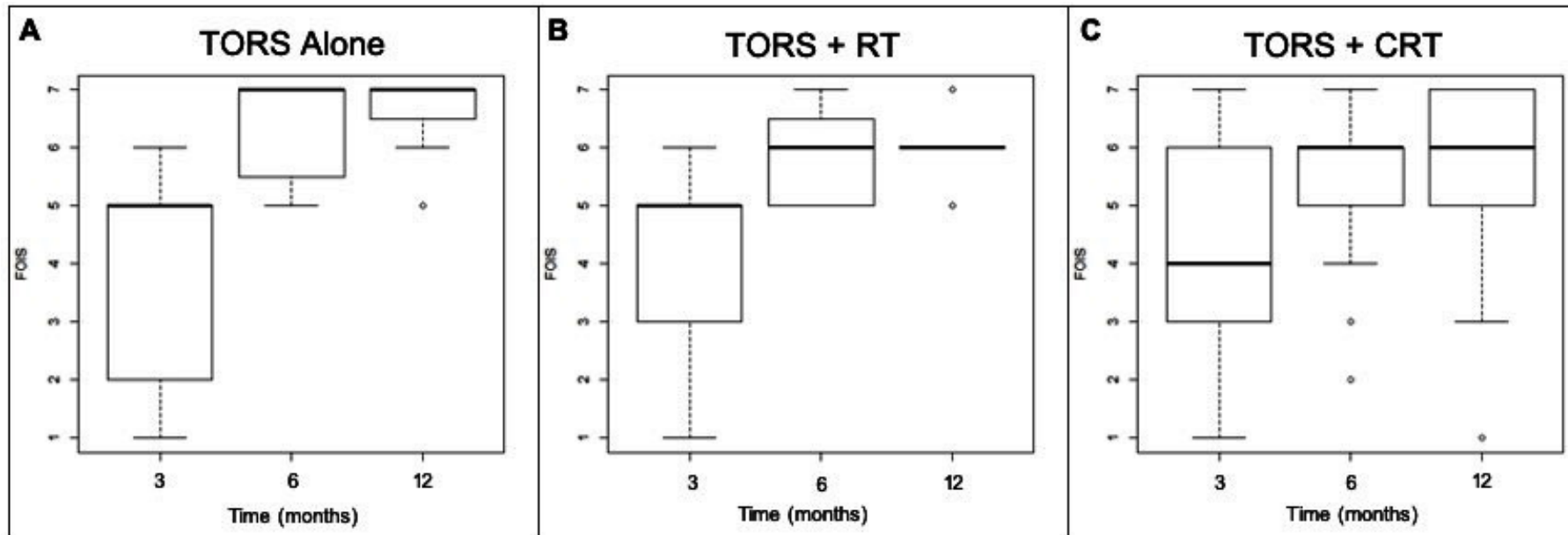

Figure 2. Boxplot diagram demonstrating FOIS swallowing results at 3, 6, and 12 months among the three treatment groups. (A) represents the TORS alone treatment group, (B) represents the TORS+RT treatment group, and (C) represents the TORS+CRT treatment group. CRT, chemoradiation; FOIS, functional oral intake scale; RT, radiation therapy; TORS, transoral robotic surgery.

strated normal or near-normal oral intake at 6 and 12 months post-treatment, with median FOIS scores in all groups between 6 and 7 at 6 and 12 months post-treatment. Table 3 shows the median and interquartile ranges of FOIS scores at 3, 6, and 12 months post-treatment in these three subgroups in a descriptive format.

\section{FOIS Scores: 3 Months Post-Treatment}

At 3 months post-treatment, the TORS alone and the TORS+RT groups had similar FOIS results with a median score of 5 (Figure 2A-2B, Table 3). The TORS+CRT group had slightly lower FOIS results with a median score of 4 (Figure 2C). All treatment groups had subjects who were percutaneous endoscopic gastrostomy (PEG) tube dependent with no statistically significant differences between groups $(P=0.500)$.

\section{FOIS Scores: 6 Months Post-Treatment}

At 6 months post-treatment, all groups demonstrated an improvement in median FOIS score (Figure 2A-2C, Table 3). Three subjects in the TORS+CRT group continued to be PEG tube dependent at 6 months. All other subjects were tolerating oral intake with FOIS scores greater than or equal to 4 . In the TORS alone group, the median FOIS score reached 7 , indicating a return to normal oral intake without restrictions (Figure 2A, Table 3).

\section{FOIS Scores: 12 Months Post-Treatment}

At 12 months post-treatment, the median FOIS scores remained unchanged from the scores at 6-month post-treatment in all groups (Figure $2 \mathrm{~A}-2 \mathrm{C}$, Table 3). However, the lower end of the interquartile range for TORS alone and TORS+RT groups increased from 5.5 to 6.5 and 5 to 6 , respectively (Figure 2A2B). This demonstrates an improved swallowing function to near-normal FOIS scores.

There were no statistically significant differences observed in swallowing assessment through FOIS scores among each of the subgroups at 3, 6, or 12 months of follow-up. When comparing swallowing assessment through median FOIS scores of the TORS group to the TORS plus chemoradiation group at 12 months however, a statistically significant difference was nearly reached $(\mathrm{P}=0.055)$.

\section{DISCUSSION}

The incidence of OPSCC is predicted to continue to rise over the next several years [3]. As HPV-related OPSCC affects a younger patient population and por- tends a favorable overall prognosis secondary to improved response to treatment, function-sparing treatment is the paramount goal in this population $[16,28,30]$. Swallowing dysfunction, or dysphagia, is one of the most untoward treatment effects in this population and can significantly impact quality of life [27,36-38]. Radiation treatment in this population damages normal tissues as well as decreases salivary flow and increases connective tissue scarring [3941]. Postoperative chemotherapy has been found to independently predict for prolonged gastrostomy after TORS and has significant toxicity and adverse functional effects, dysphagia among the most commonly cited impairments [42-46]. Understanding the functional impact of adjuvant therapy in this patient population is of great importance.

Examining functional outcomes in head and neck SCC TORS patients using the FOIS has been previously demonstrated in the literature. Genden et al. compared FOIS scores in head and neck SCC patients that underwent either primary CRT or TORS plus adjuvant therapy. The primary CRT group showed reduced FOIS compared to the TORS plus adjuvant therapy group in both the short- and long-term post-treatment [33]. Additionally, Van Adel et al. examined FOIS scores in TORS+RT and TORS+CRT after surgical intervention and immediately post-treatment and found the number of patients tolerating a total oral diet without restrictions decreased following adjuvant therapy [47]. While these studies have similarly used the FOIS to examine functional outcomes in TORS and are in line with our findings, our study is the first of its kind to compare TORS alone, TORS+RT, and TORS+CRT in the post-treatment period.

In this study, we assessed the functional swallowing outcomes in the HPV-related OPSCC TORS patient population. The FOIS scale was used as a measure of swallowing outcome, as it provides useful information regarding dysphagia-based oral food and liquid intake, from which the clinician can extrapolate meaningful conclusions [31]. For example, we can understand that taking in a diet of a single consistency or even one of multiple consistencies requiring special preparation or compensations can impact quality of life by affecting relationship with others, comfortability with going out to eat or performing compensatory tasks in public [48-49]. We compared FOIS scores among three treatment groups (TORS alone, TORS+RT, and TORS+CRT) at 3 months, 6 months, and 12 months. The 3-month time point represented short-term swallowing function while the 12-month time point represented long-term swallowing function.

Swallowing function was most impacted in the short term. At this time, the TORS alone and TORS+RT patient cohorts were tolerating a total diet of multiple consistencies but requiring special preparation or compensations. However, in the TORS+CRT cohort, the swallowing function was more adversely affected, with the average patient only taking an oral diet of single consistency 


\section{ORIGINAL}

\begin{tabular}{|c|c|c|c|c|}
\hline Variable & Total & TORS & TORS + RT & TORS + CRT \\
\hline Sample size & 60 & 11 & 12 & 37 \\
\hline Age & 63 [57.75-66.25] & $63[60.5-67.5]$ & $63.5[59.5-67]$ & 62 [57-66] \\
\hline \multicolumn{5}{|l|}{ Gender } \\
\hline Male & $43(72)$ & $5(45)$ & $8(67)$ & $30(81)$ \\
\hline Female & $17(28)$ & $6(55)$ & $4(33)$ & $7(19)$ \\
\hline \multicolumn{5}{|l|}{ Race } \\
\hline Black & $3(5)$ & $0(0)$ & 1 (8) & $2(5)$ \\
\hline Hispanic & $10(17)$ & $3(27)$ & 1 (8) & $6(16)$ \\
\hline White & $46(77)$ & $8(73)$ & $9(75)$ & $29(78)$ \\
\hline Asian & $0(0)$ & $0(0)$ & $1(8)$ & $0(0)$ \\
\hline Other & 1 (2) & $0(0)$ & $0(0)$ & $0(0)$ \\
\hline \multicolumn{5}{|l|}{ Smoking } \\
\hline Yes & $27(45)$ & $5(45)$ & $5(42)$ & $17(46)$ \\
\hline No & $33(55)$ & $6(55)$ & $7(58)$ & $20(54)$ \\
\hline \multicolumn{5}{|l|}{ pT stage } \\
\hline 1 & $26(43)$ & $5(45)$ & $3(25)$ & $18(49)$ \\
\hline 2 & $30(50)$ & $6(55)$ & $8(67)$ & $16(43)$ \\
\hline 3 & $3(5)$ & $0(0)$ & $1(8)$ & $2(5)$ \\
\hline 4 & $1(2)$ & $0(0)$ & $0(0)$ & 1 (3) \\
\hline \multicolumn{5}{|l|}{ pN stage } \\
\hline 0 & $7(12)$ & $4(36)$ & $2(17)$ & 1 (3) \\
\hline 1 & $51(85)$ & $7(64)$ & $10(83)$ & $34(92)$ \\
\hline 2 & $2(3)$ & $0(0)$ & $0(0)$ & $2(5)$ \\
\hline \multicolumn{5}{|l|}{ M stage } \\
\hline 0 & $60(100)$ & $11(100)$ & $12(100)$ & $37(100)$ \\
\hline$x$ & $0(0)$ & $0(0)$ & $0(0)$ & $0(0)$ \\
\hline \multicolumn{5}{|l|}{ AJCC stage } \\
\hline 1 & $54(90)$ & $11(100)$ & $11(92)$ & $32(87)$ \\
\hline 2 & $5(8)$ & $0(0)$ & 1 (8) & $4(11)$ \\
\hline 3 & $1(2)$ & $0(0)$ & $0(0)$ & 1 (3) \\
\hline \multicolumn{5}{|c|}{ PEG placement } \\
\hline Yes & $27(45)$ & $4(36)$ & $4(33)$ & $19(51)$ \\
\hline No & $33(55)$ & $7(64)$ & $8(67)$ & $18(49)$ \\
\hline
\end{tabular}

Data are presented as $\mathrm{n}(\%)$ or median [interquartile range]. AJCC stage is based on pathologic staging. AJCC, American Joint Committee on Cancer; CRT, chemoradiation; PEG, percutaneous endoscopic gastrostomy; RT, radiation therapy; TORS, transoral robotic surgery.

at 3 months. Prior studies have shown that the intensity of chemotherapy has an acute additive adverse impact on swallowing function, largely due to a higher incidence of mucositis which can linger for months after treatment [28]. Our observations mirror this study and highlight the acute functional sequelae of triple therapy.

At 6 months, the intermediate time point, the patients who underwent adjuvant treatment were tolerating a diet of multiple consistencies without special preparation but with specific food limitations whereas the average swallowing function in the TORS alone subgroup had improved to a level likely comparable to pre-treatment swallowing function. In line with a previous study by More et al., very few patients were dependent on the use of PEG tube at 6-month follow-up [40].

Interestingly, the swallowing function reached at the 6-month time point in our study predicted the long-term (12 months) swallowing outcome in each of the individual treatment groups. Although all three treatment groups improved in swallowing function over time, the addition of postoperative adjuvant radiation and chemoradiation treatment appeared to prevent some patients from attaining a diet of total oral intake without restrictions by the 12-month time point. While these results do not reach statistical significance, we are supported by previous studies which have shown that relative to TORS alone, FOIS scores were lower and dysphagia much more debilitating in the patients who underwent postoperative adjuvant treatment $[16,25]$.

There is great variability within the literature in the documentation of dysphagia symptoms and their functional impact on TORS patients after treatment for OPSCC. The most commonly utilized swallowing outcome measurement is PEG tube dependence [28]. In a review of this subject, Sinclair et 


\begin{tabular}{llll}
\hline Table 3. FOIS Scores After Treatment & & & \\
\hline Group & 3 months & 6 months & 12 months \\
\hline TORS & $5[2-5]$ & $7[5.5-7]$ & $7[6.5-7]$ \\
\hline TORS + RT & $5[3-5]$ & $6[5-6.25]$ & $6[6-6]$ \\
\hline TORS + CRT & $4[3-6]$ & $6[5-6]$ & $6[5-7]$ \\
\hline
\end{tabular}

Data are presented as median [interquartile range]. CRT, chemoradiation, FOIS, functional oral intake scale; RT, radiation therapy; TORS, transoral robotic surgery.

al. discussed several prior studies that have measured functional outcomes following TORS for oropharyngeal cancer using PEG tube retention and the need for tracheostomy as primary end points. However, as few patients require tracheostomies or retain PEG tubes, these outcome measures do not provide an adequate assessment of swallowing function [50]. Some studies have assessed dietary outcomes using the standardized Performance Status Scale-Head \& Neck questionnaires to make conclusions regarding the impact of cancer treatment on swallow function [29]. Others utilize the MD Anderson Dysphagia Inventory or video fluoroscopic imaging $[16,29,51]$. We chose to use the FOIS as a measure of swallowing function because it can be easily applied in a clinician setting, gives a standardized subjective understanding of the patient's oral intake, and can be implemented in the follow-up care of OPSCC patients after TORS. Interpretation of our FOIS scores has allowed us to make conclusions that, though not statistically significant, have great clinical relevance. FOIS is a simple and convenient tool that can be used reliably to monitor improvement in short term and long-term functional swallowing outcomes in patients with OPSCC after TORS with or without adjuvant treatment.

Several limitations are inherent in our study design. Our small sample size among the three treatment groups led to a low-powered statistical analysis which precluded us from making broad conclusions. Furthermore, a large number of patients were excluded from the study due to the lack of records available at the specific 3-, 6-, and 12-month time points. Due to the retrospective nature of the study and small sample size, our three study groups are not homogenous and may exhibit selection bias. Having a better understanding of the patient's swallowing status with a baseline swallowing evaluation in the pre-treatment period would also aid in this type of analysis. Since the time of the study, our standard of care has evolved such that we now incorporate pre-treatment swallowing evaluations to better understand any baseline limitations and subsequent impact of various interventions. Future studies to compare pre- and post-treatment swallowing function and following these subjects for longer follow-up periods are necessary to further discern the significance of our results.

\section{CONCLUSION}

Dysphagia is an important consequence of cancer treatment and has overarching implications on quality of life. Using the FOIS, we demonstrated that swallowing function may be worse in the long term in patients with OPSCC undergoing triple therapy, although this finding did not reach statistical significance. This study emphasizes the importance of diligent selection in patients undergoing TORS to avoid poor functional swallowing outcomes, particularly in those that may need adjuvant chemoradiation therapy. A study with a larger sample size may determine the significance of these trends.

\section{ARTICLE INFORMATION}

*Correspondence: Caitlin Coviello, MBA, Don Soffer Clinical Research Center, 1120 NW 14th Street, $5^{\text {th }}$ Floor Miami, Florida 33136, USA. Email: cmc367@med.miami.edu

Received: Nov. 27, 2019; Accepted: Feb. 24, 2020; Published: Apr. 24, 2020

DOI: $10.24983 /$ scitemed.aohns.2020.00127
Acknowledgments: This manuscript has not been previously published and is not under consideration in the same or substantially similar form in any other peer-reviewed media. All of the authors listed have contributed sufficiently to the project to be included as authors, and no additional acknowledgements are relevant. Caitlin Coviello, Dr. Opeoluwa Daniyan, and Dr. Giovana Thomas had full access to all of the data in the study and take responsibility for the integrity of the data and the accuracy of the data analysis.

Presentations: Podium presentation at the Triological Society Combined Sections Meeting, January 24-26, 2019 in Coronado, CA, USA; and poster presentation at the Florida Society of Otolaryngologists Combined Meeting November 8-10, 2019 in West Palm Beach, FL, USA.

Ethics Approval and Consent to Participate: The study is in accordance with the ethical standards of the 1964 Helsinki declaration and its later amendments or comparable ethical standards.

Funding: University of Miami Department of Otolaryngology-Head \& Neck Surgery.

Conflict of Interest: No actual or potential conflicts of interest exist.

Copyright @ 2020 The Authors. This is an open-access article distributed under the terms of the Creative Commons Attribution 4.0 International License (CC-BY).

\section{REFERENCES}

1. Chaturvedi AK, Engels EA, Pfeiffer RM, et al. Human papillomavirus and rising oropharyngeal cancer incidence in the United States. J Clin Oncol 2011;29(32):4294-4301.

2. Pytynia KB, Dahlstrom KR, Sturgis EM. Epidemiology of HPV-associated oropharyngeal cancer. Oral Oncol 2014;50(5):380-386.

3. $\mathrm{Xu} \mathrm{L,} \mathrm{Dahlstrom} \mathrm{KR,} \mathrm{Lairson} \mathrm{DR,} \mathrm{Sturgis} \mathrm{EM.} \mathrm{Projected} \mathrm{oropharyngeal} \mathrm{carcinoma}$ incidence among middle-aged US men. Head Neck 2019;41(9):3226-3234.

4. Taberna M, Mena M, Pavon MA, Alemany L, Gillison ML, Mesia R. Human papillomavirus-related oropharyngeal cancer. Ann Oncol 2017;28(10):2386-2398.

5. Davidson J, Freeman J, Birt D. Mandibulotomy in the irradiated patient. Arch Otolaryngol Head Neck Surg 1989;115(4):497-499.

6. Shaha AR. Mandibulotomy and mandibulectomy in difficult tumors of the base of the tongue and oropharynx. Semin Surg Oncol 1991;7(1):25-30.

7. Shah JP, Gil Z. Current concepts in management of oral cancer--surgery. Oral Oncol 2009;45(4-5):394-401.

8. Kamran SC, Qureshi MM, Jalisi S, Salama A, Grillone G, Truong MT. Primary surgery versus primary radiation-based treatment for locally advanced oropharyngeal cancer. Laryngoscope 2018;128(6):1353-1364.

9. Hirano M, Kuroiwa Y, Tanaka S, Matsuoka H, Sato K, Yoshida T. Dysphagia following various degrees of surgical resection for oral cancer. Ann Otol Rhinol Laryngol 1992;101(2 Pt 1):138-141.

10. Pauloski BR, Rademaker AW, Logemann JA, et al. Surgical variables affecting swallowing in patients treated for oral/oropharyngeal cancer. Head Neck 2004;26(7):625-636.

11. Pauloski BR, Rademaker AW, Logemann JA, et al. Pretreatment swallowing function in patients with head and neck cancer. Head Neck 2000;22(5):474-482.

12. Starmer HM, Tippett D, Webster $K$, et al. Swallowing outcomes in patients with oropharyngeal cancer undergoing organ-preservation treatment. Head Neck 2014;36(10):1392-1397.

13. Schache AG, Lieger O, Rogers P, Kelly A, Newman L, Kalavrezos N. Predictors of swallowing outcome in patients treated with surgery and radiotherapy for advanced oral and oropharyngeal cancer. Oral Oncol 2009;45(9):803-808.

14. Weinstein GS, O'Malley BW, Jr., Cohen MA, Quon H. Transoral robotic sur- 
gery for advanced oropharyngeal carcinoma. Arch Otolaryngol Head Neck Surg 2010;136(11):1079-1085.

15. Paleri V, Fox H, Winter S. Transoral Robotic Surgery for Oropharyngeal Cancer ORL J Otorhinolaryngol Relat Spec 2018;80(3-4):156-170.

16. Leonhardt FD, Quon H, Abrahao M, O'Malley BW, Jr., Weinstein GS. Transoral robotic surgery for oropharyngeal carcinoma and its impact on patient-reported quality of life and function. Head Neck 2012;34(2):146-154.

17. Moore EJ, Henstrom DK, Olsen KD, Kasperbauer JL, McGree ME. Transoral resec tion of tonsillar squamous cell carcinoma. Laryngoscope 2009;119(3):508-515.

18. Moore EJ, Olsen KD, Kasperbauer JL. Transoral robotic surgery for oropharyngeal squamous cell carcinoma: a prospective study of feasibility and functional outcomes. Laryngoscope 2009;119(11):2156-2164.

19. Moore EJ, Olsen SM, Laborde RR, et al. Long-term functional and oncologic results of transoral robotic surgery for oropharyngeal squamous cell carcinoma. Mayo Clin Proc 2012;87(3):219-225.

20. Weinstein GS, O'Malley BW, Jr., Snyder W, Sherman E, Quon H. Transoral robotic surgery: radical tonsillectomy. Arch Otolaryngol Head Neck Surg 2007;133(12):1220-1226.

21. Lorincz BB, Mockelmann N, Busch CJ, Knecht R. Functional outcomes, feasibility, and safety of resection of transoral robotic surgery: single-institution series of 35 consecutive cases of transoral robotic surgery for oropharyngeal squamous cell carcinoma. Head Neck 2015;37(11):1618-1624.

22. Varma VR, Eskander A, Kang SY, et al. Predictors of gastrostomy tube dependence in surgically managed oropharyngeal squamous cell carcinoma. Laryngoscope 2019;129(2):415-421.

23. Berman TA, Schiller JT. Human papillomavirus in cervical cancer and oropharyngeal cancer: one cause, two diseases. Cancer 2017;123(12):2219-2229.

24. Evans M, Newcombe R, Fiander A, et al. Human papillomavirus-associated oropharyngeal cancer: an observational study of diagnosis, prevalence and prog nosis in a UK population. BMC Cancer 2013;13:220

25. Duek I, Billan S, Amit M, Gil Z. Transoral Robotic Surgery in the HPV Era. Rambam Maimonides Med J 2014;5(2):e0010.

26. Weinstein GS, Quon H, Newman HJ, et al. Transoral robotic surgery alone for oropharyngeal cancer: an analysis of local control. Arch Otolaryngol Head Neck Surg 2012;138(7):628-634.

27. Chen MM, Roman SA, Kraus DH, Sosa JA, Judson BL. Transoral Robotic Surgery: a population-level analysis. Otolaryngol Head Neck Surg 2014;150(6):968-975.

28. Masterson L, Moualed D, Liu ZW, et al. De-escalation treatment protocols for human papillomavirus-associated oropharyngeal squamous cell carcinoma: a systematic review and meta-analysis of current clinical trials. Eur J Cancer 2014;50(15):2636-2648.

29. Chen AY, Frankowski R, Bishop-Leone J, et al. The development and validation of a dysphagia-specific quality-of-life questionnaire for patients with head and neck cancer: the M. D. Anderson dysphagia inventory. Arch Otolaryngol Head Neck Surg 2001;127(7):870-876.

30. Hutcheson KA, Holsinger FC, Kupferman ME, Lewin JS. Functional outcomes after TORS for oropharyngeal cancer: a systematic review. Eur Arch Otorhinolaryngol 2015;272(2):463-471.

31. Crary MA, Mann GD, Groher ME. Initial psychometric assessment of a functional oral intake scale for dysphagia in stroke patients. Arch Phys Med Rehabil 2005;86(8):1516-1520.

32. van der Molen L, van Rossum MA, Ackerstaff AH, Smeele LE, Rasch CRN, Hilgers FJM. Pretreatment organ function in patients with advanced head and neck cancer: clinical outcome measures and patients' views. BMC Ear, Nose and Throat Disorders 2009;9(1):10.

33. Genden EM, Kotz T, Tong CC, et al. Transoral robotic resection and reconstruc tion for head and neck cancer. Laryngoscope 2011;121(8):1668-1674.

34. American Joint Committee on Cancer. AJCC cancer staging form supplement to the AJCC cancer staging manual, eighth edition.

35. National Comprehensive Cancer Network. NCCN clinical practice guidelines in oncology: head and neck cancers version 1.2018.

36. Hoxbroe Michaelsen S, Gronhoj C, Hoxbroe Michaelsen J, Friborg J, von Buchwald C. Quality of life in survivors of oropharyngeal cancer: a systematic review and meta-analysis of 1366 patients. Eur J Cancer 2017;78:91-102.

37. Raber-Durlacher JE, Brennan MT, Verdonck-de Leeuw IM, et al. Swallowing dysfunction in cancer patients. Supportive Care in Cancer 2012;20(3):433-443.

38. Jones E, Speyer R, Kertscher B, Denman D, Swan K, Cordier R. Health-related quality of life and oropharyngeal dysphagia: a systematic review. Dysphagia 2018;33(2):141-172.

39. Sonis ST. Oral mucositis in head and neck cancer: risk, biology, and management. Am Soc Clin Oncol Educ Book 2013.

40. More Yl, Tsue TT, Girod DA, et al. Functional swallowing outcomes following transoral robotic surgery vs primary chemoradiotherapy in patients with advanced-stage oropharynx and supraglottis cancers. JAMA Otolaryngology-Head \& Neck Surgery 2013;139(1):43-48.

41. Trotti A, Bellm LA, Epstein JB, et al. Mucositis incidence, severity and associated outcomes in patients with head and neck cancer receiving radiotherapy with or without chemotherapy: a systematic literature review. Radiother Oncol 2003;66(3):253-262.

42. Hunter KU, Lee OE, Lyden TH, et al. Aspiration pneumonia after chemo-intensity-modulated radiation therapy of oropharyngeal carcinoma and its clinical and dysphagia-related predictors. Head Neck 2014;36(1):120-125.

43. Nguyen NP, Smith HJ, Sallah S. Evaluation and management of swallowing dysfunction following chemoradiation for head and neck cancer. Curr Opin Otolaryngol Head Neck Surg 2007;15(2):130-133.

44. Machtay M, Moughan J, Trotti A, et al. Factors associated with severe late toxicity after concurrent chemoradiation for locally advanced head and neck cancer: an RTOG analysis. J Clin Oncol 2008;26(21):3582-3589.

45. Pohar S, Demarcantonio M, Whiting P, Crandley E, Wadsworth J, Karakla D. Percutaneous endoscopic gastrostomy tube dependence following chemoradiation in head and neck cancer patients. Laryngoscope 2015;125(6):1366-1371.

46. Terrell JE, Ronis DL, Fowler KE, et al. Clinical predictors of quality of life in patients with head and neck cancer. Arch Otolaryngol Head Neck Surg 2004;130(4):401408.

47. Van Abel KM, Quick MH, Graner DE, et al. Outcomes following TORS for HPV-positive oropharyngeal carcinoma: PEGs, tracheostomies, and beyond. Am J Otolaryngol 2019;40(5):729-734.

48. Ekberg O, Hamdy S, Woisard V, Wuttge-Hannig A, Ortega P. Social and psychological burden of dysphagia: its impact on diagnosis and treatment. Dysphagia 2002;17(2):139-146.

49. Nazarko L. The management of dysphagia in palliative care. Int J Palliat Nurs 2017;23(4):162-164.

50. Sinclair CF, McColloch NL, Carroll WR, Rosenthal EL, Desmond RA, Magnuson JS. Patient-perceived and objective functional outcomes following transoral robotic surgery for early oropharyngeal carcinoma. Arch Otolaryngol Head Neck Surg 2011;137(11):1112-1116.

51. Ursino S, Seccia V, Cocuzza P, et al. How does radiotherapy impact swallowing function in nasopharynx and oropharynx cancer? Short-term results of a prospective study. Acta Otorhinolaryngol Ital 2016;36(3):174-184. 